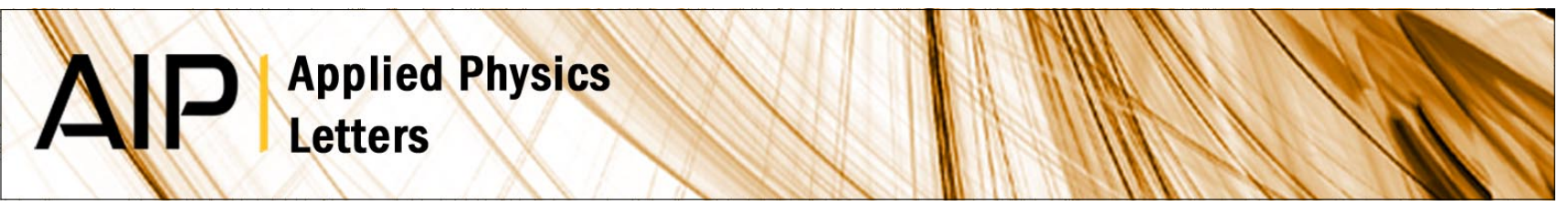

\title{
Temporal and spatial evolution of the electronic density and temperature of the plasma produced by laser ablation of $\mathrm{LiNbO} 3$
}

\section{F. J. Gordillo-Vázquez, A. Perea, J. A. Chaos, J. Gonzalo, and C. N. Afonso}

Citation: Appl. Phys. Lett. 78, 7 (2001); doi: 10.1063/1.1335853

View online: http://dx.doi.org/10.1063/1.1335853

View Table of Contents: http://apl.aip.org/resource/1/APPLAB/v78/i1

Published by the American Institute of Physics.

\section{Related Articles}

A high-resolution integrated model of the National Ignition Campaign cryogenic layered experiments Phys. Plasmas 19, 056315 (2012)

Crossed-beam energy transfer in direct-drive implosions

Phys. Plasmas 19, 056314 (2012)

Surface processing technique based on opto-hydrodynamic phenomena occurring in laser-induced breakdown of a microdroplet

Appl. Phys. Lett. 100, 104104 (2012)

Production and acceleration of ion beams by laser ablation

Rev. Sci. Instrum. 83, 02B717 (2012)

Proton emission from a laser ion source

Rev. Sci. Instrum. 83, 02B310 (2012)

\section{Additional information on Appl. Phys. Lett.}

Journal Homepage: http://apl.aip.org/

Journal Information: http://apl.aip.org/about/about_the_journal

Top downloads: http://apl.aip.org/features/most_downloaded

Information for Authors: http://apl.aip.org/authors

\section{ADVERTISEMENT}

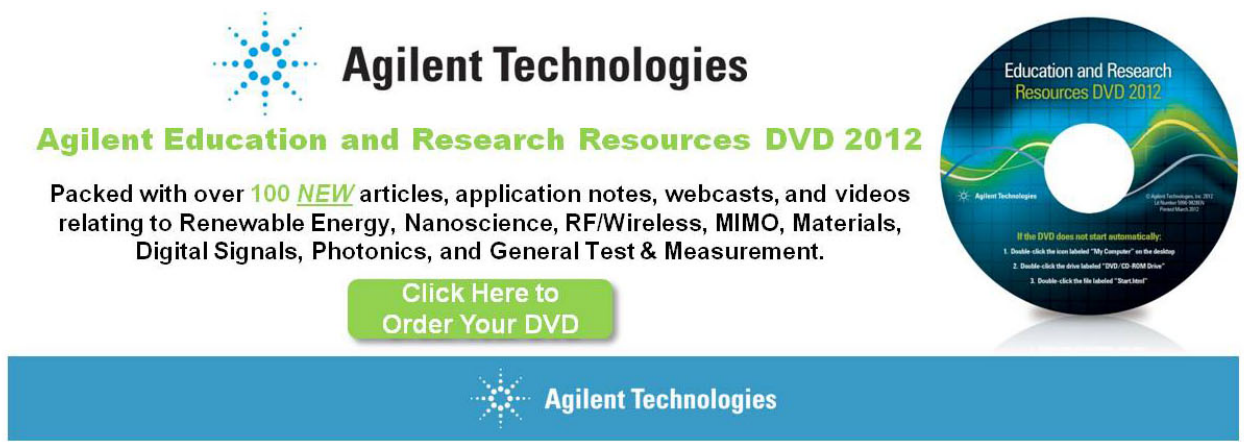




\title{
Temporal and spatial evolution of the electronic density and temperature of the plasma produced by laser ablation of $\mathrm{LiNbO}_{3}$
}

\author{
F. J. Gordillo-Vázquez ${ }^{\text {a) }}$ \\ Instituto de Ciencia de Materiales de Madrid, C.S.I.C., Cantoblanco, 28049 Madrid, Spain
}

\author{
A. Perea, J. A. Chaos, J. Gonzalo, and C. N. Afonso \\ Instituto de Óptica, C.S.I.C., Serrano 121, 28006 Madrid, Spain
}

(Received 17 July 2000; accepted for publication 30 October 2000)

\begin{abstract}
Emission spectroscopy is used to determine the spatial and temporal evolution of the electronic temperature $\left(T_{e}\right)$ and density $\left(N_{e}\right)$ of the plasma generated by laser ablation in vacuum of a $\mathrm{LiNbO}_{3}$ target. It is found that whereas $N_{e}$ (in the range of $10^{16} \mathrm{~cm}^{-3}$ ) decreases by a factor of 2 as the distance to the target surface increases $10 \mathrm{~mm}, T_{e}$ (in the range $0.7-0.8 \mathrm{eV}$ ) only decreases $10 \%$ between 2 and $4 \mathrm{~mm}$ from the target to remain nearly constant for longer distances. While $T_{e}$ is almost constant in time at the studied distances, $N_{e}$ presents a maximum at a time delay that increases when increasing the distance to the target surface. The space-time constancy of $T_{e}$ is related to a collisional decoupling of the heavy species in the plasma for distances longer than 2-4 mm. (C) 2001 American Institute of Physics. [DOI: 10.1063/1.1335853]
\end{abstract}

Laser ablation has become a very promising tool for both advanced micromachining and thin-film deposition. In most of the cases, a plasma formed by neutral and ionized species is produced whose kinetics has been widely studied in order to understand the physical mechanisms controlling the thin-film deposition process and properties. ${ }^{1}$ Nevertheless, a very limited number of works have been focused on the study of fundamental plasma parameters, such as electron temperature and electron density, or the nature of the dominant plasma excitation processes in different spatial and temporal regions. $^{2-5}$ Although they are essential to evaluate the energy transport into plasma as well as to understand plasma reactivity, such studies have mostly been performed on metals. $^{2,3}$

Lithium niobate $\left(\mathrm{LiNbO}_{3}\right)$ is a well-known material with excellent nonlinear properties of interest for many applications that normally require the production of high-quality thin films. ${ }^{6}$ Although pulsed-laser deposition (PLD) has proven to be one of the most successful techniques in growing complex oxide materials, ${ }^{7}$ the films deposited by ablation of $\mathrm{LiNbO}_{3}$ are often found to be Li deficient. ${ }^{8,9}$ Nevertheless, the studies on the desorption processes ${ }^{10}$ or the plasma kinetics $^{11-13}$ are very limited. The aim of this work is to determine the electron density $\left(N_{e}\right)$ and the electron temperature $\left(T_{e}\right)$ of the plasma produced by laser ablation of a $\mathrm{LiNbO}_{3}$ single crystal as a function of the time delay after the laser pulse and the distance to the target surface. The relative emission line intensities of successive ionization stages of $\mathrm{Nb}$ atoms are used for determining $T_{e}$ while Starkbroadened profiles of neutral $\mathrm{Li}$ atoms are used for quantifying $N_{e} \cdot{ }^{14-16}$

Estimation of $N_{e}$ using Stark broadening of spectral line profiles of an isolated atom or singly charged ions is a wellestablished and reliable technique. ${ }^{14-16}$ In vacuum, both

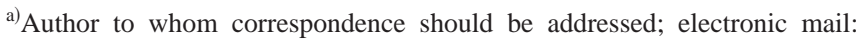
vazquez@io.cfmac.csic.es
}

Doppler and Stark broadening can contribute to spectral line broadening. The effect due to Doppler broadening $[\sim 0.12 \AA$ at full width at half maximum (FWHM)] can be neglected when compared to the Stark broadening resulting from collisions with charged particles that dominate for plasma densities above $10^{14} \mathrm{~cm}^{-3}$. ${ }^{16}$ Stark broadening of well-isolated lines from neutral atoms is predominantly caused by electron impact, the FWHM of the Stark-broadened lines, $\Delta \lambda_{1 / 2}$, being related to $N_{e}\left(\right.$ in $\left.\mathrm{cm}^{-3}\right)$ by ${ }^{16}$

$$
\Delta \lambda_{1 / 2}=2 W\left[\frac{N_{e}}{10^{16}}\right] \AA
$$

where $W$ is an electron impact parameter which is a weak function of the temperature and only changes by a factor less than 2 over the temperature range $10000-80000 \mathrm{~K}$. In the case of the LiI transition considered in this work, the 610.3 nm doublet $\left(3 d^{2} D_{3 / 2,5 / 2} \rightarrow 2 p^{2} P_{1 / 2,3 / 2}\right)$, we have used $W$ $=0.216 \AA$ (for $T_{e}=10000 \mathrm{~K}$ ) to determine $N_{e} \cdot{ }^{15,16}$

The plasma was generated by ablation of a $\mathrm{LiNbO}_{3}$ single crystal using an ArF excimer laser with a FWHM pulse duration $\cong 20 \mathrm{~ns}$ and a repetition rate of $5 \mathrm{~Hz}$. The target was mounted in a rotating holder and placed in a vacuum chamber evacuated to a residual pressure of 6.0 $\times 10^{-7}$ mbar. The angle of incidence of the laser beam was $45^{\circ}$, and the laser energy density at the target surface was 1.2 $\mathrm{J} \mathrm{cm}^{-2}$ (irradiance $\sim 0.06 \mathrm{GW} \mathrm{cm}^{-2}$ ), this energy being the one typically used to grow films. ${ }^{8}$ The plasma was $\times 2$ imaged onto the $30 \mu \mathrm{m}$ entrance slit of a $\frac{3}{4} \mathrm{~m}$ monochromator with a maximum resolution of $0.05 \mathrm{~nm}$. This image was scanned along the normal to the target with a spatial resolution of $0.18 \mathrm{~mm}$ as reported elsewhere. ${ }^{13}$ The light was finally collected by a photomultiplier ( $15 \mathrm{~ns}$ rise time) connected to a boxcar averager/gated integrator or to a digitizer. The results presented here start at a distance of $2 \mathrm{~mm}$ to the target to be sure that the contribution from the continuum can be neglected. ${ }^{17}$ 


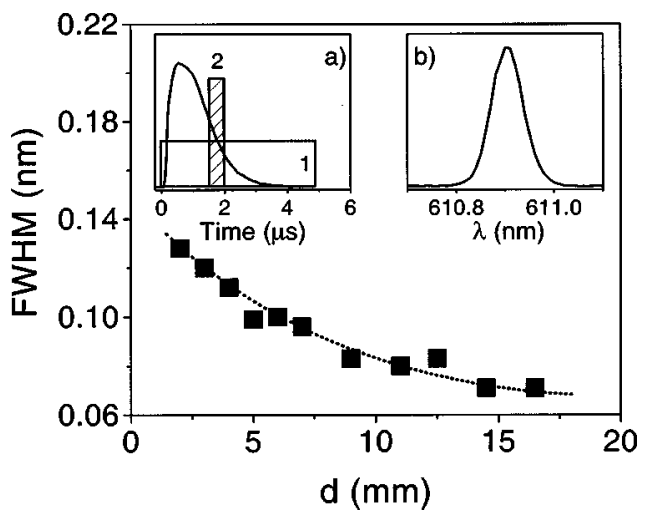

FIG. 1. FWHM of the LiI $610.3 \mathrm{~nm}$ line as a function of the distance to the target surface using the time-integrated configuration. Inset (a) shows the duration of a typical emission transient and the two gate widths used in this work. Inset (b) shows a Stark-broadened line measured at $7 \mathrm{~mm}$ from the target. The dashed line is a guide for the eye.

Inset (a) in Fig. 1 shows a typical emission transient in which the two gate widths of the boxcar used are shown: its value is wide enough as to contain the whole emission transient (gate 1, time-integrated signal) or small compared to the duration of the transient (50 ns, gate 2, time-resolved signal). Both signals were collected when scanning the wavelength at each distance. The Stark-broadened profile of the LiI line measured at $7 \mathrm{~mm}$ is shown in inset (b). The time-integrated FWHM of the Stark-broadened LiI transition, $\Delta \lambda_{1 / 2}$, is found to decrease as the distance to the target was increased, as seen in Fig. 1.

Figure 2 shows the time-integrated $N_{e}$ as a function of the distance to the target surface calculated through Eq. (1) from the experimental results plotted in Fig. 1. The $N_{e}$ values are found to first decrease rapidly, then remain practically constant. The dashed line is a $1 / d$ fit of the experimental data. This space dependence is very similar to that reported earlier for plasmas produced in graphite ${ }^{4}$ and $\mathrm{YBa}_{2} \mathrm{Cu}_{3} \mathrm{O}_{7}$ (Ref. 5) for similar space intervals, though much higher-power density levels were used in those works. The time-resolved values of $N_{e}$ for different distances to the target surface were evaluated through similar measurements but in time-resolved configuration. The results are shown in the inset where it is seen that $N_{e}$ first increases up to a maximum, then decreases (following a $1 / t^{2}$ law already noted by

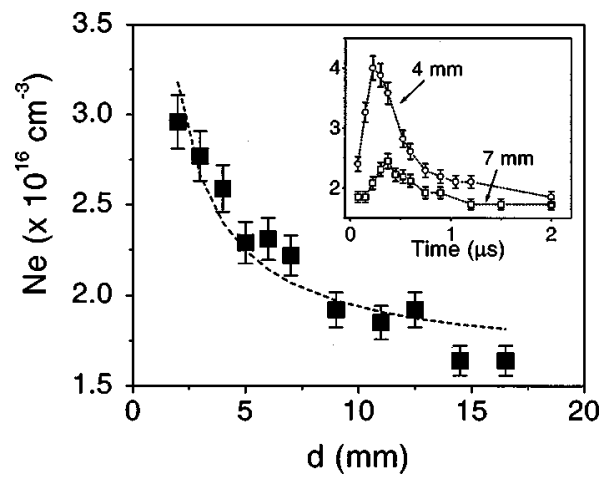

FIG. 2. Time-integrated $N_{e}(\mathbf{\square})$ as a function of the distance to the target. The dashed line shows a $1 / d$ fit of the experimental data. The inset shows the time-resolved evolution of $N_{e}$ at different distances to the target: $4 \mathrm{~mm}$ (○) and $7 \mathrm{~mm}(\square)$. The vertical axis in the inset also refers to $N_{e}$ (in $\left.10^{16} \mathrm{~cm}^{-3}\right)$. others $^{2,4}$ ) during $\approx 700 \mathrm{~ns}$ to a nearly constant value, this maximum only observed before in the ablation of metals. ${ }^{18}$ This dependence together with the time position of the maximum reminds us very much that of the ground-state $\mathrm{Li}$ atom density measured in plasmas produced by laser ablation of $\mathrm{Li}$ targets $^{19}$ and the LiI emission transients (whose shape is somehow related to the $\mathrm{Li}$ atom density) produced by ablation of $\mathrm{LiNbO}_{3}$ measured both in this work [Fig. 1(a)] and in an earlier one. ${ }^{13}$

Accurate determination of $T_{e}$ is done by using the ratio of the two line intensities from successive ionization stages of the same element. Assuming local thermodynamic equilibrium (LTE), the ratio of such line intensities is given by ${ }^{16}$

$$
\begin{aligned}
\frac{I_{1}}{I_{2}}= & \left(\frac{f_{1} g_{1} \lambda_{2}^{3}}{f_{2} g_{2} \lambda_{1}^{3}}\right)\left(4 \pi^{3 / 2} a_{0}^{3} N_{e}\right)^{-1}\left(\frac{k T_{e}}{E_{H}}\right)^{3 / 2} \\
& \times \exp \left(\frac{-E_{1}+E_{2}-E_{\infty}+\Delta E_{\infty}}{k T_{e}}\right),
\end{aligned}
$$

where $I_{1}$ represents the line of the atom with a higher ionization degree, $f_{1,2}$ are the absorption oscillator strengths, $g_{1,2}$ are the statistical weights (or degeneracies of the states), $a_{0}$ is the Bohr radius, $E_{H}$ is the ionization energy of the hydrogen atom, $E_{1,2}$ are the excitation energy, and $\Delta E_{\infty}$ is the correction to the ionization energy $E_{\infty}$ of the lower ionization stage due to plasma interactions. For high-density plasmas, the correction factor in the ionization energy is given by ${ }^{14}$

$$
\Delta E_{\infty}(\mathrm{eV})=3 z b \frac{e^{2}}{4 \pi \varepsilon_{0}}\left[\frac{4 \pi 10^{6} N_{e}}{3}\right]^{1 / 3},
$$

where $z=2$ for the lowest ionization state, $e$ is the electron charge (in $\mathrm{C}$ ), $\varepsilon_{0}$ (in $\mathrm{Fm}^{-1}$ ) is the permittivity of the free space, $N_{e}$ is in $\mathrm{cm}^{-3}$, and $b=6.2414 \times 10^{18}$ is the energy conversion factor from joules to $\mathrm{eV}$. In order to determine $T_{e}$, the NbII $478.9 \mathrm{~nm}$ and $\mathrm{NbI} 407.97 \mathrm{~nm}$ lines were measured following the same procedure as in the case of the LiI line illustrated in Fig. 1. The spectroscopic constants for the two $\mathrm{Nb}$ lines were taken from Ref. 20. An optical depth of $\tau \cong 0.5$ for the $407.97 \mathrm{~nm}$ line was calculated according to Ref. 15, assuming thermal equilibrium for the lower state density $\left(\sim 7 \times 10^{14} \mathrm{~cm}^{-3}\right)$ of the $407.97 \mathrm{~nm}$ transition of $\mathrm{NbI}$, a plasma length of $0.1 \mathrm{~cm}$ and a ionization degree of $10 \%$. The $\tau$ found could have some impact on the opacity; however, we assumed an optically thin plasma as a first approximation.

Figure 3 shows the dependence of the time-integrated $T_{e}$ on the distance to the target surface, whereas the inset shows its temporal evolution. Near the target $T_{e}$ slightly decreases when increasing the distance, whereas it is almost constant in time, contrary to what has been reported for plasmas produced by laser ablation of metals, ${ }^{2,3}$ graphite, ${ }^{4}$ and $\mathrm{YBa}_{2} \mathrm{Cu}_{3} \mathrm{O}_{7} .{ }^{5}$ We have assumed the validity of LTE that is valid provided that electron-heavy particle (atom, ion, or molecule) collisions are very fast and dominate the radiative processes. A necessary (though not sufficient) condition for having LTE is that $N_{e}\left(\mathrm{~cm}^{-3}\right) \geqslant 1.4 \times 10^{14} \times T_{e}^{1 / 2} \times(\Delta E) .{ }^{3} \mathrm{In}$ our case, for the transition $407.97 \mathrm{~nm}$ of $\mathrm{NbI}$ with $\Delta E$ $=3.03 \mathrm{eV}$ and $T_{e}=0.8 \mathrm{eV}$, we have that LTE might start to hold for $N_{e} \geqslant 3.5 \times 10^{15} \mathrm{~cm}^{-3}$, which is lower than the values 


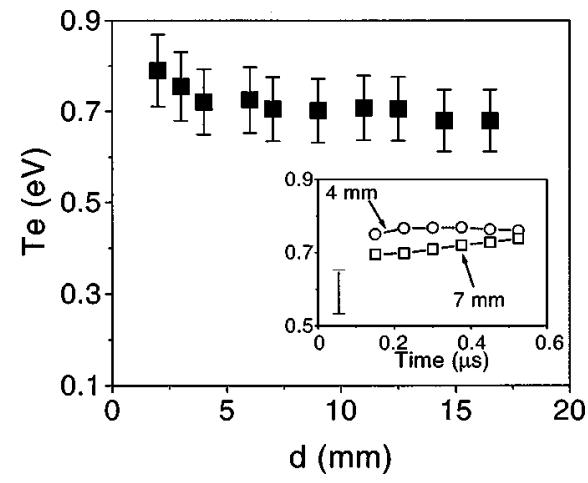

FIG. 3. Time-integrated $T_{e}(\boldsymbol{\square})$ as a function of the distance to the target. The inset shows the time-resolved evolution of $T_{e}$ at different distances from the target: $4 \mathrm{~mm}(\bigcirc)$ and $7 \mathrm{~mm}(\square)$. The vertical axis in the inset also refers to $T_{e}$ (in $\left.\mathrm{eV}\right)$. The relative error in the inset is shown as an error bar.

we determined. Moreover, in a transient plasma like ours, LTE is accepted to exist if the time between collisions (here, $\tau_{\mathrm{ei}} \sim 2 \mathrm{ps}$ ) of the species is small (as in our plasma) compared with the time scale of any significant change in the plasma. ${ }^{21}$

The values of $N_{e}\left(1.5-4.0 \times 10^{16} \mathrm{~cm}^{-3}\right)$ and $T_{e}(0.7-0.8$ $\mathrm{eV})$ determined here are not high enough for significant absorption by inverse bremsstrahlung. For distances where strong inverse bremsstrahlung occurs (hundreds of $\mu \mathrm{m}$ ) densities in the range $10^{19}-10^{18} \mathrm{~cm}^{-3}$ have been reported. ${ }^{22}$ Our results are consistent with the fact that the contribution of the continuum emission becomes, typically, negligible for distances longer than $2 \mathrm{~mm} .{ }^{17}$ The usually reported decrease of $N_{e}$ with time and distance ${ }^{2-5}$ is related to the free expansion from a point source into a large volume. The 1/d law fit of $N_{e}$ at short distances indicates good agreement with the predictions of the plume expansion model proposed earlier. ${ }^{23}$

The most striking result when comparing the present study to earlier ones relates to $T_{e}$. In particular to the fact that it remains nearly constant both in time and distance within the studied intervals (Fig. 3). A weak dependence of $T_{e}$ on time (but stronger than that observed in the present work) has been reported for times longer than $300 \mathrm{~ns}$ and related to the fact that the cooling due to the expansion process becomes, in part, compensated by the energy released by the recombinations. ${ }^{4}$ A more likely explanation might be found by taking into account the fact that the species in the plasma have slightly different velocities which make them separate from each other at longer distances/times. If this happens, the atom-atom collision probability, and thus the cooling of the plasma, decreases. The constant $T_{e}$ calculated from the line intensity of successive ionization stages of $\mathrm{Nb}$ atoms, assuming LTE, means that the temperature of $\mathrm{Nb}$ ions and neutrals is similar to that of the electrons. It is thus consistent with an atom-atom collisionless expansion of $\mathrm{Nb}$ atoms for distances longer than $2 \mathrm{~mm}$. The long-lasting delayed release of Li atoms with high velocities due to an electronic mechanism (most likely involving electron ejection) reported elsewhere ${ }^{12}$ should contribute further to the observed time-space constancy of $T_{e}$. Nevertheless, whether this constancy is a general conclusion for plasmas generated by ablation of insulators in which defects play an essential role in the ablation process or it is related to the special kinetics of the $\mathrm{LiNbO}_{3}$ plasma reported elsewhere ${ }^{12,13}$ is not yet clear and needs further investigation.

In conclusion, the spatial and temporal evolutions of $T_{e}$ and $N_{e}$ in the plasma produced by laser ablation of $\mathrm{LiNbO}_{3}$ have successfully been determined through the line intensities of two successive ionization states of $\mathrm{Nb}$ and the Starkbroadened profiles of a $\mathrm{Li}$ line. In the target neighborhood, $N_{e}$ follows a $1 / d$ dependence on the distance $d$ to the target surface, consistent with a one-dimensional expansion, while $T_{e}$ shows little dependence on $d$. The time evolution of $N_{e}$ exhibits a maximum value that occurs at longer times for larger distances. Moreover, $T_{e}$ is found to be almost constant both in time and space, and thus it is concluded that the plasma expansion is atom-atom collisionless for distances longer than $2 \mathrm{~mm}$.

This work was partially funded by CICYT (Spain), under Project No. TIC99-0866. Two of the authors (J.A.C. and F.J.G.V.) acknowledge a grant and a contract from the Spanish Ministery of Education and Culture.

${ }^{1}$ Pulsed Laser Deposition, edited by D. B. Chrisey and G. K. Hubler (Wiley, New York, 1994).

${ }^{2}$ J. M. Hendron, C. M. O. Mahony, T. Morrow, and W. G. Graham, J. Appl. Phys. 81, 2131 (1997).

${ }^{3}$ B. Toftmann, J. Schou, T. N. Hansen, and J. G. Lunney, Phys. Rev. Lett. 84, 3998 (2000).

${ }^{4}$ S. S. Harilal, C. V. Bindhu, R. C. Issac, V. P. N. Nampoori, and C. P. G. Vallabhan, J. Appl. Phys. 82, 2140 (1997).

${ }^{5}$ S. S. Harilal, C. V. Bindhu, V. P. N. Nampoori, and C. P. G. Vallabhan, Appl. Spectrosc. 52, 449 (1998).

${ }^{6}$ Properties of Lithium Niobate, EMIS Datareviews Series No. 5 (IEEE, London, 1989).

${ }^{7}$ C. N. Afonso, in Materials for Optoelectronics: New Developments, edited by F. Agulló-López (World Scientific, Singapore, 1995), Chap. 1, and references therein.

${ }^{8}$ S. B. Ogale, R. Nawathey-Dikshit, S. J. Dikshit, and S. M. Kanetkar, J. Appl. Phys. 71, 5718 (1992).

${ }^{9}$ Y. Shibata, Y. Kanno, K. Kaya, M. Knai, and T. Kawai, Jpn. J. Appl. Phys., Part 1 34, L320 (1995).

${ }^{10}$ M. Affatigato, K. Tang, R. F. Haglund, Jr., and C. H. Chen, Appl. Phys. Lett. 65, 1751 (1994).

${ }^{11}$ Th. Beuermann, H. J. Brinkmann, T. Damm, and M. Stuke, Mater. Res. Soc. Symp. Proc. 191, 37 (1990).

${ }^{12}$ J. A. Chaos, R. W. Dreyfus, A. Perea, R. Serna, J. Gonzalo, and C. N. Afonso, Appl. Phys. Lett. 76, 649 (2000).

${ }^{13}$ C. N. Afonso, F. Vega, J. Gonzalo, and C. Zaldo, Appl. Surf. Sci. 69, 149 (1993).

${ }^{14}$ H. R. Griem, Spectral Line Broadening by Plasmas (Academic, New York, 1974)

${ }^{15}$ H. R. Griem, Principles of Plasma Spectroscopy (Cambridge University Press, Cambridge, U.K., 1997).

${ }^{16}$ G. Bekefy, Principles of Laser Plasmas (Wiley, New York, 1976).

${ }^{17}$ J. Gonzalo, F. Vega, and C. N. Afonso, J. Appl. Phys. 77, 6588 (1995).

${ }^{18}$ I. Weaver, G. W. Martin, W. G. Graham, T. Morrow, and C. L. S. Lewis, Rev. Sci. Instrum. 70, 1801 (1999).

${ }^{19}$ M. Harnafi and B. Dubreuil, J. Appl. Phys. 69, 7565 (1991).

${ }^{20}$ C. H. Corliss and W. R. Bozman, Experimental Transition Probabilities for Spectral Lines of Seventy Elements, NBS Monograph Vol. 53 (NBS, Washington, DC, 1962).

${ }^{21}$ Y. B. Zeldovich and Y. P. Raizer, Physics of Shock Waves and HighTemperature Hydrodynamic Phenomena (Academic, New York, 1966).

${ }^{22}$ A. Al-Khateeb, L. A. Doyle, A. H. El-Astal, M. J. Lamb, C. L. S. Lewis, G. W. Martin, T. Morrow, G. J. Pert, D. Riley, I. Weaver, and T. P. Williamson, Appl. Phys. A: Mater. Sci. Process. 69, S479 (1999).

${ }^{23}$ R. K. Singh and J. Narayan, Phys. Rev. B 41, 8843 (1990). 